\title{
Dexpramipexole shows promise for ALS in phase II trial
}

The putative mitochondrial modulator dexpramipexole seems to slow functional decline and might reduce mortality in patients with amyotrophic lateral sclerosis (ALS), according to a phase II study.

ALS is a fatal neurodegenerative disease, for which riluzole is the sole approved treatment and only extends patient survival by a matter of months. A previous open-label study had suggested a trend towards attenuated functional decline in patients treated with dexpramipexole.

The current trial, which was published in Nature Medicine, comprised two parts and enrolled only patients who had been diagnosed with ALS within the past 24 months. "The value of this enrolment criterion was that the trial included a high proportion of patients that were declining rapidly, increasing the potential to detect a drug effect over a short time course," says Valentin Gribkoff, the corresponding author of the study.

In part one, the safety and tolerability of the drug were assessed. 102 patients were randomly assigned to receive placebo or daily oral doses of $50 \mathrm{mg}, 150 \mathrm{mg}$ or $300 \mathrm{mg}$ dexpramipexole for 12 weeks. Two patients in the $300 \mathrm{mg}$ group experienced reversible neutropenia, but no deaths or treatment-related serious adverse events occurred.

In part two of the study, after a 4-week, single-blind placebo washout phase, patients were randomly assigned to daily doses of $50 \mathrm{mg}$ or $300 \mathrm{mg}$ dexpramipexole for 24 weeks. "The unique study design enabled the amount of time patients spent on placebo to be minimized, which is an important consideration given the rapid course of the disease," says Gribkoff. The aim of part two

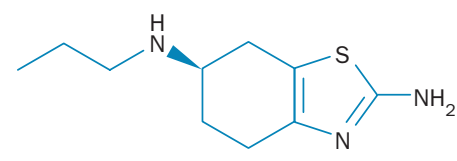

The chemical structure of dexpramipexole.

was to perform a preliminary assessment of drug efficacy, with primary outcomes of mortality and rate of functional decline, as classified on the ALS Functional Rating Scale-Revised (ALSFRS-R).

Patients in the high-dose group experienced a $20 \%$ reduction in the rate of decline of the ASLFRS-R score relative to those in the low-dose group. Moreover, deaths occurred in nine patients in the $50 \mathrm{mg}$ group, and only three patients in the $300 \mathrm{mg}$ group. These effects were not statistically significant, however, owing to the small patient numbers -92 individuals in part two.

The researchers carried out an additional statistical analysis to account for the effect of patient deaths on the group ALSFRS-R scores. "Patients with the worst scores usually die first, and once they are removed from the analysis the mean level of that group artificially improves," explains Gribkoff. In a Joint Rank analysis, patients who died early were ranked lowest, and those who experienced the smallest ALSFRS-R decline were ranked highest. The results of this analysis showed a statistically significant benefit of $300 \mathrm{mg}$ dexpramipexole. "This represents the first evidence of a drug-related decrease in functional decline in a well-controlled ALS trial," says Gribkoff. A large phase III trial of the drug is currently underway.

Katie Kingwell

Original article Cudkowicz, M. et al. The effects of dexpramipexole (KNS-760704) in individuals with amyotrophic lateral sclerosis. Nat. Med. doi:10.1038/nm.2579 